\title{
Association of Educational Interventions with Clinician Learning and Ventilator- Associated Pneumonia Patient Outcomes: A Protocol for Systematic Review and Meta-Analysis
}

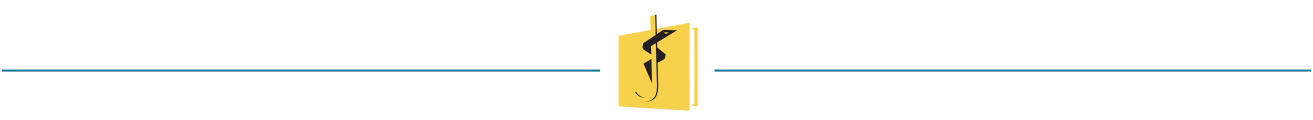

\author{
John Mark M. Gutiérrez, RN, MA, PhD (candidate) 1,2,3* \\ Allan L. Dueño, RN, MSN, MIH (candidate) ${ }^{4,5}$ \\ Arnel E. Sonido, $P h D, R M T, R N, R M^{4}$ \\ Jerry A. Abriam, $P h D, R N, R M^{6}$ \\ Emmanuel D. Paragas, Jr., DNS, RN ${ }^{7}$ \\ Alain Jason A. Generale, CCRC, DRDM, MAN, \\ MHSS, $R N^{8}$
}

\section{ABSTRACT}

Recent studies have shown that educational interventions for ventilator-associated pneumonia (VAP) prevention may result in positive outcomes in intensive care units. However, other studies investigating this kind of intervention have produced inconsistent results. Thus this paper reports a protocol for systematic

John Mark M. Gutiérrez

jmgutierrez@su.edu.sa

Divisions of Nursing Research and Evidence-Based Practice, Department of Nursing

2 Division of Epidemiology, Department of Clinical Laboratory Science, College of Applied Medical Sciences, Shaqra University, Riyadh, Saudi Arabia

3 School of Advanced Studies, Saint Louis University, Baguio, Philippines

4 Intensive Care Unit, Lorma Medical Center, San Fernando, Philippines
\end{abstract}

review and planned meta-analysis to investigate the association of instituted VAP educational interventions with clinician learning and patient outcomes. In this review, the authors will identify relevant citations from electronic databases, reference lists, and other sources; screen articles against predetermined eligibility criteria; appraise each study using the Cochrane Collaboration's risk of bias assessment

5 University of the Philippines Open University, Los Baños, Philippines

6 Institute of Community Health and Allied Medical Sciences, Don Mariano Marcos Memorial State University, San Fernando, Philippines

7 Department of Nursing, West Liberty University, West Liberty, USA

8 Clinical Research Support Office, Children's Hospital Los Angeles, Los Angeles, USA 
tools and combine acquired evidence using the meta-analytic approach. The results of this review are crucial to assist clinicians and policy-makers in making well-informed decisions regarding VAP prevention practices for mechanically ventilated patients. This review protocol followed the Preferred Reporting Items for Systematic reviews and Meta-Analyses for Protocols 2015 guidelines and was registered with PROSPERO as CRD42016051561.

Keywords: clinical outcomes, educational intervention, learning outcomes, review protocol, VAP prevention, ventilator-associated pneumonia

\section{INTRODUCTION}

The rapid advances in the fields of intensive and critical care have seen VAP as a priority clinical concern for infection control and prevention programs. VAP is a type of healthcare-associated infection that develops following endotracheal intubation or mechanical ventilation in patients without pre-existing pneumonia on admission. This condition is an important cause of greater morbidity and mortality in the intensive and critical-care settings with substantial impact on healthcare costs. [1-2] Thus, measures to control or prevent its occurrence, especially during the first 96 hours of mechanical ventilatory support, are commonly advocated.

To date, the clinical practice recommendations for the prevention and management of VAP are centered on evidence-based strategies directed to prevent the risk of oropharyngeal aspiration including the build-up of pathogens in the respiratory and digestive tracts, and minimize endotracheal tube and mechanical ventilator utilization.[3-4] Other VAP prevention strategies have also been acknowledged in the literature, including simple and cost-effective measures.[5]

Evidently, one of the recognized cost-effective quality improvement strategies in low-resource intensive care units (ICUs) is the educational intervention. [6] Educational programs that include well-planned evidence-based practice elements to improve both clinician learning and patient outcomes have also become a common practice in multidisciplinary ICUs. [7-9] Over the years, there has been an increasing interest in the use of educational interventions as primary tools to prevent nosocomial pneumonia in mechanically ventilated patients. Recent studies have shown that VAP educational interventions, as the core of learning among health practitioners and professionals have resulted in positive learning and clinical outcomes.[10] Other studies investigating this kind of intervention, however, have produced inconsistent results between pre- and post-assessments, often demonstrating no significant improvements in ventilator bundle compliance.[1 1-12] VAP incidence, $[11,13-14]$ and other patient clinical outcomes.[11-15] These variations between studies inevitably suggest the need for a systematic review to address clinical uncertainties that are associated with VAP educational interventions; hence, this investigation.

The present review generally attempts to identify, critically appraise, and summarize the best available evidence to address the discordance between the results of studies using systematic review and meta-analysis of association. The review authors specifically aim to investigate whether or not administering educational intervention for VAP prevention is associated with positive outcomes that are beneficial to both the clinicians (learning outcomes) and the mechanically ventilated patients (clinical outcomes). Such an approach is considered essential to guide healthcare practitioners as well as policy-makers in making well-informed decisions regarding infection control and prevention practices for patients requiring mechanical ventilatory support.

\section{METHODS}

\section{Design}

As part of our a priori efforts in promoting transparency, this paper reports a protocol for systematic review and planned meta-analysis to investigate whether or not the institution of education-based VAP prevention intervention is associated with positive clinician learning and patient outcomes in intensive and critical care units. This review protocol was based on an established methodology [16] and followed the Preferred Reporting Items for Systematic reviews and Meta-Analyses for Protocols (PRISMA-P) 2015 guidelines (Table 1) [17-18]. In addition, this review protocol was registered with the International Prospective Register of Systematic Reviews (PROSPERO) as CRD42016051561 on November 16, 2016. The latest revisions of the protocol were made on May 26, 2017. 
Table 1. The PRISMA-P 2015 checklist

\begin{tabular}{|c|c|c|c|c|}
\hline \multirow{2}{*}{$\begin{array}{l}\text { Section/topic } \\
\text { Administrative } \\
\text { information }\end{array}$} & \multirow[t]{2}{*}{$\#$} & \multirow[t]{2}{*}{ Checklist item } & \multicolumn{2}{|c|}{ Information reported } \\
\hline & & & Yes & No \\
\hline \multicolumn{5}{|l|}{ Title } \\
\hline Identification & la & Identify the report as a protocol of a systematic review & {$[\mathrm{x}]$} & {[]} \\
\hline Update & $1 b$ & $\begin{array}{l}\text { If the protocol is for an update of a previous systematic review, } \\
\text { identify as such }\end{array}$ & [ ] & {$[x] N / A$} \\
\hline Registration & 2 & $\begin{array}{l}\text { If registered, provide the name of the registry (eg, PROSPERO) } \\
\text { and registration number in the abstract }\end{array}$ & {$[x]$} & [] \\
\hline \multicolumn{5}{|l|}{ Authors } \\
\hline Contact & $3 a$ & $\begin{array}{l}\text { Provide name, institutional affiliation, and e-mail address of all } \\
\text { protocol authors; provide the physical mailing address of the } \\
\text { corresponding author }\end{array}$ & {$[x]$} & {[]} \\
\hline Contributions & $3 b$ & $\begin{array}{l}\text { Describe contributions of protocol authors and identify the } \\
\text { guarantor of the review }\end{array}$ & {$[x]$} & {[]} \\
\hline Amendments & 4 & $\begin{array}{l}\text { If the protocol represents an amendment of a previously com- } \\
\text { pleted or published protocol, identify as such and list changes; } \\
\text { otherwise, state plan for documenting important protocol } \\
\text { amendments }\end{array}$ & {[]} & {$[x] N / A$} \\
\hline \multicolumn{5}{|l|}{ Support } \\
\hline Sources & $5 a$ & Indicate sources of financial or other support for the review & [ ] & {$[x] N / A$} \\
\hline Sponsor & $5 b$ & Provide name for the review funder and/or sponsor & [ ] & {$[x] N / A$} \\
\hline $\begin{array}{c}\text { Role of } \\
\text { sponsor/funder }\end{array}$ & $5 c$ & $\begin{array}{l}\text { Describe roles of funder(s), sponsor(s), and/or institution(s), if } \\
\text { any, in developing the protocol }\end{array}$ & {[]} & {$[x] N / A$} \\
\hline \multicolumn{5}{|l|}{ Introduction } \\
\hline Rationale & 6 & $\begin{array}{l}\text { Describe the rationale for the review in the context of what is } \\
\text { already known }\end{array}$ & {$[\mathrm{x}]$} & [ ] \\
\hline Objectives & 7 & $\begin{array}{l}\text { Provide an explicit statement of the question(s) the review will } \\
\text { address with reference to participants, interventions, compara- } \\
\text { tors, and outcomes (PICO) }\end{array}$ & {$[x]$} & [ ] \\
\hline \multicolumn{5}{|l|}{ Methods } \\
\hline $\begin{array}{l}\text { Eligibility } \\
\text { criteria }\end{array}$ & 8 & $\begin{array}{l}\text { Specify the study characteristics (eg, PICO, study design, } \\
\text { setting, time frame) and report characteristics (eg, years con- } \\
\text { sidered, language, publication status) to be used as criteria for } \\
\text { eligibility for the review }\end{array}$ & {$[x]$} & [ ] \\
\hline $\begin{array}{l}\text { Information } \\
\text { sources }\end{array}$ & 9 & $\begin{array}{l}\text { Describe all intended information sources (eg, electronic data- } \\
\text { bases, contact with study authors, trial registers, or other grey } \\
\text { literature sources) with planned dates of coverage }\end{array}$ & {$[x]$} & [ ] \\
\hline $\begin{array}{l}\text { Search } \\
\text { strategy }\end{array}$ & 10 & $\begin{array}{l}\text { The present draft of the search strategy to be used for at least } \\
\text { one electronic database, including planned limits, such that it } \\
\text { could be repeated }\end{array}$ & {$[x]$} & {[]} \\
\hline \multicolumn{5}{|l|}{ Study records } \\
\hline $\begin{array}{c}\text { Data } \\
\text { management }\end{array}$ & $11 a$ & $\begin{array}{l}\text { Describe the mechanism(s) that will be used to manage records } \\
\text { and data throughout the review }\end{array}$ & {$[x]$} & {[]} \\
\hline $\begin{array}{l}\text { Selection } \\
\text { process }\end{array}$ & $11 b$ & $\begin{array}{l}\text { State the process that will be used for selecting studies (eg, two } \\
\text { independent reviewers) through each phase of the review (ie, } \\
\text { screening, eligibility, and inclusion in meta-analysis) }\end{array}$ & {$[x]$} & {[]} \\
\hline $\begin{array}{l}\text { Data collec- } \\
\text { tion process }\end{array}$ & $11 c$ & $\begin{array}{l}\text { Describe the planned method of extracting data from reports } \\
\text { (eg, piloting forms, done independently, in duplicate), any } \\
\text { processes for obtaining and confirming data from investigators }\end{array}$ & {$[x]$} & {[]} \\
\hline Data items & 12 & $\begin{array}{l}\text { List and define all variables for which data will be sought (eg, } \\
\text { PICO items, funding sources), any pre-planned data assumptions } \\
\text { and simplifications }\end{array}$ & {$[x]$} & {[]} \\
\hline
\end{tabular}


Table 1. Continued

\begin{tabular}{|c|c|c|c|c|}
\hline \multirow{2}{*}{$\begin{array}{l}\text { Section/topic } \\
\text { Administrative } \\
\text { information }\end{array}$} & \multirow[t]{2}{*}{ \# } & \multirow[t]{2}{*}{ Checklist item } & \multicolumn{2}{|c|}{ Information reported } \\
\hline & & & Yes & No \\
\hline $\begin{array}{l}\text { Outcomes } \\
\text { and prioritization }\end{array}$ & 13 & $\begin{array}{l}\text { List and define all outcomes for which data will be sought, } \\
\text { including prioritization of main and additional outcomes, with } \\
\text { rationale }\end{array}$ & {$[x]$} & {[]} \\
\hline $\begin{array}{l}\text { Risk of bias } \\
\text { in individual } \\
\text { studies }\end{array}$ & 14 & $\begin{array}{l}\text { Describe anticipated methods for assessing the risk of bias of } \\
\text { individual studies, including whether this will be done at the } \\
\text { outcome or study level, or both; state how this information will } \\
\text { be used in data synthesis }\end{array}$ & {$[x]$} & {[]} \\
\hline \multicolumn{5}{|l|}{$\begin{array}{l}\text { Data } \\
\text { synthesis }\end{array}$} \\
\hline & $15 a$ & $\begin{array}{l}\text { Describe criteria under which study data will be quantitatively } \\
\text { synthesized }\end{array}$ & {$[\mathrm{x}]$} & {[]} \\
\hline & $15 b$ & $\begin{array}{l}\text { If data are appropriate for quantitative synthesis, describe } \\
\text { the planned summary measures, methods of handling data, } \\
\text { and methods of combining data from studies, including any } \\
\text { planned exploration of consistency (eg, }\left.\right|^{2}, \text { Kendall's tau) }\end{array}$ & {$[x]$} & {[]} \\
\hline & $15 c$ & $\begin{array}{l}\text { Describe any proposed additional analyses (eg, sensitivity or } \\
\text { subgroup analyses, meta-regression) }\end{array}$ & {$[x]$} & {[]} \\
\hline Meta-bias(es) & 16 & $\begin{array}{l}\text { Specify any planned assessment of meta-bias(es) (eg, publica- } \\
\text { tion bias across studies, selective reporting within studies) }\end{array}$ & {$[\mathrm{x}]$} & {[]} \\
\hline $\begin{array}{l}\text { Confidence } \\
\text { in cumulative } \\
\text { evidence }\end{array}$ & 17 & $\begin{array}{l}\text { Describe how the strength of the body of evidence will be } \\
\text { assessed (eg, GRADE) }\end{array}$ & {$[x]$} & [ ] \\
\hline
\end{tabular}

GRADE, the Grading of Recommendations Assessment, Development and Evaluation; N/A, not applicable; PICO, Population Intervention Comparison Outcome; PRISMA-P, Preferred Reporting Items for Systematic Review and Meta-Analysis Protocols.

This checklist has been adapted for use with protocol submissions to PROSPERO from Table 3 in Moher D, et al. Preferred reporting items for systematic review and meta-analysis protocols (PRISMA-P) 2015 statement. Systematic Reviews 2015 4:1

\section{Eligibility Criteria}

In compliance with the eligibility criteria, two of the review authors (EP \& AD) will independently assess each study involved. Any assessment discrepancy will be resolved by virtue of consensus or consultation with a third investigator (JMG or AJG).

\section{Types of studies}

In this review, the review authors will include published and unpublished interventional studies (randomized controlled trials or RCTs, non-experimental quasi-experimental methods, pretest-posttest studies, time series designs) or observational studies (cohort and case-control studies) with equivalent or non-equivalent control groups investigating VAP educational interventions. The studies of one group design will be considered if detailed descriptions of exposure to such interventions are adequately reported. The review authors will exclude basic researches such as animal, cell, genetic, and methodology studies, including descriptive surveys, qualitative research, case series, case reports, diagnostic accuracy trials, review articles, protocols, clinical practice guidelines, brief reports, conference proceedings, commentaries, and editorials.

\section{Types of Participants}

All ICU patients, regardless of age, requiring mechanical ventilation and receiving education-based VAP prevention strategies will be included as subjects in this review to examine patient outcomes. In addition, this review will involve all clinicians working in ICU settings as subjects to examine their learning outcomes. These clinicians may include ICU nurses, infection control practitioners, intensivists, respiratory therapists, physicians, and the like. They must be directly involved in the care of mechanically ventilated patients.

Both subjects will be classified according to exposures: exposed and non-exposed groups (controls or comparators), which are defined as those who received and did not receive VAP educational interventions, respectively. Clinicians will be considered as the non-exposed group if they practice the usual 
or routine care or have no active interventions involved. Patients labeled as non-exposed pertain to those who have not received any educational interventions for the VAP prevention program.

Excluded in this review are the non-ICU or non-medical healthcare staff members, as well as, the patients who received mechanical ventilation for less than 24 hours.

\section{Types of Interventions/Exposures}

The present review applies to any type of education-based infection control interventions for VAP prevention. These may include formal or non-formal lectures, standardized sessions, use of validated selfstudy modules, fact sheets, presentations, visual aids (posters), educational handouts, educational conferences, trainings (in-service), seminars, and reinforcement at the bedside or return demonstrations that provided information on guidelines or strategies for the prevention of VAP, such as VAP bundle care or ventilator bundle and the like. The contents of such preventive practices may be based on the Centers for Disease Control and Prevention (CDC) guidelines, Institute for Healthcare Improvement (IHI) and/ or from other reputable agencies. Such educational interventions must be preceded by a pre-test (pre-exposure) and followed by a post-test (post-exposure) to determine any change in learning among the clinicians (learning outcomes) and among the patients (patient outcomes). Any non-VAP-related interventions will be excluded.

\section{Types of Outcome Measures}

The primary outcomes of this review are knowledge of and adherence to VAP educational intervention (learning outcomes), and VAP incidence (clinical outcomes). Any related VAP definitions will be accepted for inclusion in the systematic review and meta-analysis. Secondary outcomes include duration of mechanical ventilation, ICU/hospital length of stay, microbial colonization (VAP-causing microbes), cost of antibiotic treatment of VAP and hospitalization, and ICU or in-hospital mortality. Non-VAP-related outcomes will be excluded.

\section{Types of ICU Settings}

Studies conducted in intensive and critical care settings will be included. The types of ICU may include, but not limited to, combined or general ICU, coronary care unit, cardiovascular/surgical, surgical, trauma, medical, neurological/neurosurgical, surgical trauma, and burn units. The non-ICU settings will be excluded.

\section{Types of Language Use}

No language restrictions will be hereby imposed. Non-English studies will be translated accordingly for the purposes of inclusion.

\section{Timing}

No date or period restrictions will be imposed for this present review. However, studies that do not meet the above-mentioned criteria will be excluded. A summary of the review eligibility criteria is presented in Table 2.

\section{Search Methods for Identification of Studies}

Electronic searches

The review authors will search relevant studies without date and language restrictions in the following scientific databases: MEDLINE through PubMed, The Cochrane Library, Cumulative Index to Nursing and Allied Health Literature (CINAHL), Web of Science, and Education Resources Information Center (ERIC).

\section{Searching Other Resources}

Reference lists and citations of the included articles/ relevant reviews will be manually checked. Other sources leg, PROSPERO, OpenGrey, ProQuest Theses \& Dissertations, and trial registry: www. clinicaltrials.gov) of potential articles will also be explored. In addition, the review authors will utilize all possible measures to contact the corresponding authors for clarification of unpublished data results by e-mail and other provided contact information. They will also explore the authors' personal page through ResearchGate to identify other similar and/ or related studies.

\section{Searching Strategy}

Specific search strategy involving the use of Medical Subject Headings (MeSH), Boolean operators, parentheses and truncation symbols will be applied. 
Table 2. Eligibility criteria

\begin{tabular}{|c|c|}
\hline Types of study designs & $\begin{array}{l}\text { Interventional studies } \\
\text { Randomized controlled trial or clinical trial } \\
\text { Non-randomized study (quasi-experimental design) or pretest-posttest intervention } \\
\text { Observational studies } \\
\text { Cohort study } \\
\text { Case-control study }\end{array}$ \\
\hline Types of participants & $\begin{array}{l}\text { Clinicians (exposed and unexposed groups) } \\
\text { Mechanically ventilated patients (exposed and unexposed groups) }\end{array}$ \\
\hline Types of exposures & Educational interventions for VAP prevention (as defined by study authors) \\
\hline Types of outcome measures & $\begin{array}{l}\text { Any of the following outcome measures: } \\
\text { Primary outcome measures } \\
\text { Learning outcomes: } \\
\text { Knowledge (as measured by study authors) } \\
\text { Adherence (as measured by study authors) } \\
\text { Clinical outcome: } \\
\text { VAP incidence (as assessed by study authors) } \\
\text { Secondary outcome measures } \\
\text { Clinical outcomes: } \\
\text { Duration of MV (time, measured in days or equivalent) } \\
\text { ICU LOS (time, measured in days or equivalent) } \\
\text { In-hospital LOS (time, measured in days or equivalent) } \\
\text { Microbial colonization } \\
\text { Cost of antibiotic treatment of VAP (currency, converted in US dollars or equivalent) } \\
\text { Hospitalization costs (currency, converted in US dollars or equivalent) } \\
\text { ICU or in-hospital mortality (as described by study authors) }\end{array}$ \\
\hline Types of ICU settings & No restrictions imposed \\
\hline Types of languages & No restrictions imposed \\
\hline Timing & No time restrictions imposed \\
\hline
\end{tabular}

ICU, intensive care unit; LOS, length of stay; MV, mechanical ventilation; US, United States; VAP, ventilator-associated pneumonia

Table 3. MEDLINE (via PubMed) search strategy

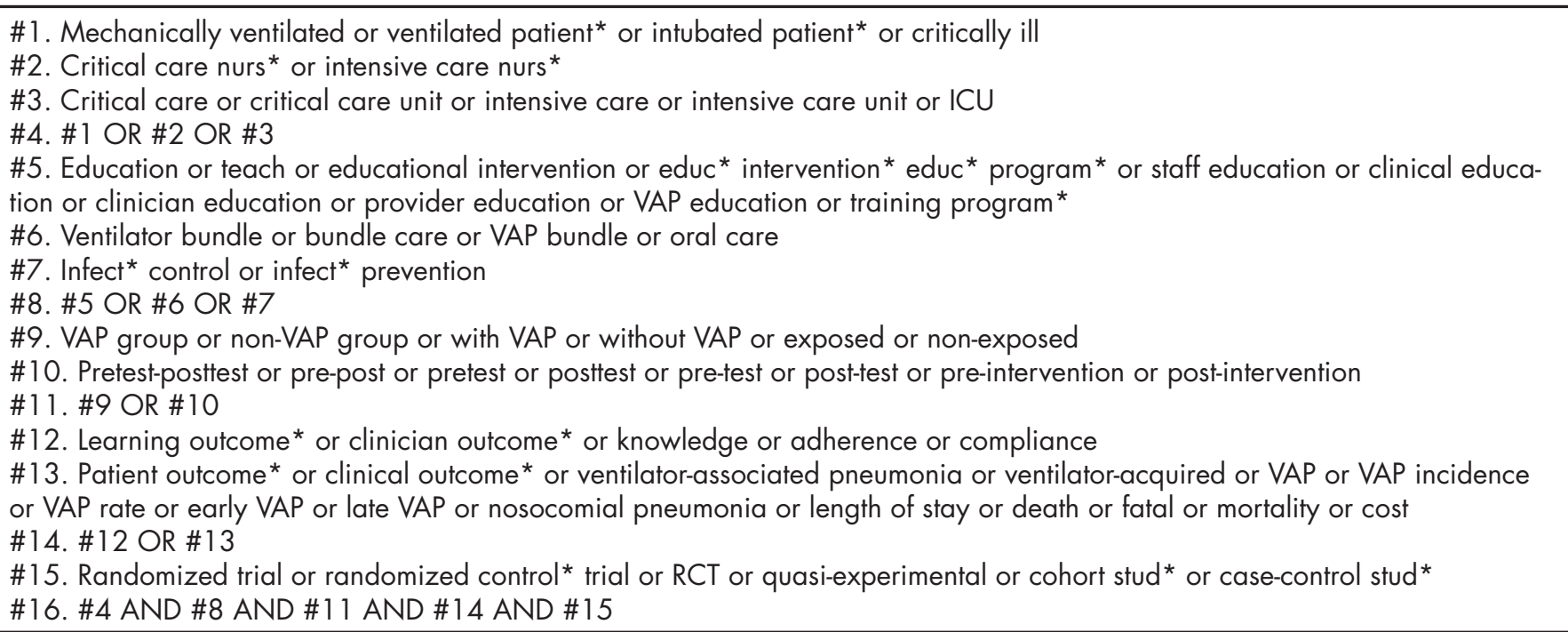

ICU, intensive care unit; RCT, randomized controlled trial; VAP, ventilator-associated pneumonia

The search strategy will be framed using the recommended modified PICO (population, intervention, comparator, outcomes) format: types of study population, exposure of interest, comparators, and outcomes or response (PECO).[16] The generated $\mathrm{MeSH}$ terms and keywords will be combined with
'OR' to give a set of results for each part of PECO, including the study design (S). Thereafter, the review authors will combine the sets of results for $P, E, C, O$, and $S$ using 'AND' to narrow the search.

A sample draft of the MEDLINE search strategy is shown in Table 3. This was developed by the first 
review author (JMG) and independently validated by the other review team members (EP, JA, AS, and $A D)$ through the above-mentioned databases. Disagreements in the design were resolved through discussions among the authors. The final version of the MEDLINE search strategy will be adapted to other databases as applicable.

\section{Data Collection and Analysis}

\section{Selection of Studies}

Two independent review authors (EP and AD) will sufficiently record the flow of information through the different phases of review. This process involves identification of relevant citations from multiple databases and other sources (reference lists, similar studies or reviews, citation tracking, unpublished manuscripts); de-duplication of search results using Microsoff ${ }^{\circledR}$ Office software, Mac Excel 2011 version; screening of titles and abstracts for relevance; retrieval of full-text articles for eligibility assessment and inclusion of potential study articles in qualitative and quantitative syntheses.

Using Microsoff ${ }^{\circledR}$ Excel, the review authors will manually transcribe the following data: first author, study title, journal name, publication date and publisher. These data will be screened for duplicates and relevance by manually searching as they automatically appear within the Excel file. Following the study selection process, the review authors will generate study codes for the included articles. For instance, the most recent included article (study 1) will be encoded as 2017-0001 (published year-assigned code). Afterward, they will use the succeeding published dates with corresponding assigned codes to other included studies leg, 2017-0002 for study 2; 2017-0003 for study 3). For multiple reports of the same study, they will allocate a single study code. A third review author (JMG or AJG) will be consulted to resolve any discordant assessments. Figure 1 illustrates the PRISMA flow diagram of study selection.[20]

\section{Quality Assessment}

In order to critically appraise the quality of included studies, the review authors will be using the Cochrane Risk of Bias Tool [19] and the ROBINS-I (Risk Of Bias In Non-randomized Studies of Interventions) [21] for RCTs and non-randomized studies (quasi-randomized, cohort and case-control studies), respectively. These tools will provide a systematic approach to organize and present the available evidence relating to the risk of bias and quality assessment.

The Cochrane Collaboration's tool for assessing the risk of bias in randomized trials covers six important domains of biases: selection bias (random sequence generation and allocation concealment), performance bias, detection bias, attrition bias, reporting bias and other biases. Within each domain, assessments are made for one or more items, which may cover different aspects of the domain or different outcomes. The bias in each of the identified domains is given the following judgment labels: "low risk" if any bias that is present is unlikely to seriously alter the results; "high risk" if the bias may alter the results seriously; or "unclear risk" if the risk of bias raises some doubt on the results. The assessments will be reported along with descriptive justifications or a summary of the pertinent characteristics of the study being evaluated to show bases of such judgment.

The ROBINS-I tool, on the other hand, covers similar important domains of biases that can be attributed to non-randomized studies. The first two are bias due to confounding and bias in the selection of participants; both cover issues during the pre-intervention phase (baseline). The third domain at the intervention phase includes bias in the classification of interventions. The four domains pertinent in the post-intervention phase include biases due to deviations from intended interventions, missing data, measurement of outcomes and selection of the reported result. The judgment of bias risk in each domain, which, in turn, affects the overall risk of bias will be facilitated by answering the signaling questions with "Yes", "Probably Yes", "No", "Probably No", or "No Information". From these questions, the domain-level or the overall risk of bias can then be identified as "low risk", "moderate risk", serious risk", "critical risk", or if the needed information is not available, "no information". Studies that will reveal being at "critical risk" or "serious risk" of bias will be excluded from the analysis, while those studies with no clear indication of biases or lacking important information will be used with caution.

All review authors will perform the above-mentioned quality assessment measures. In particular, for studies with learning outcomes, two review authors (AS \& JA) will independently assess the quality of 


\section{Identification}

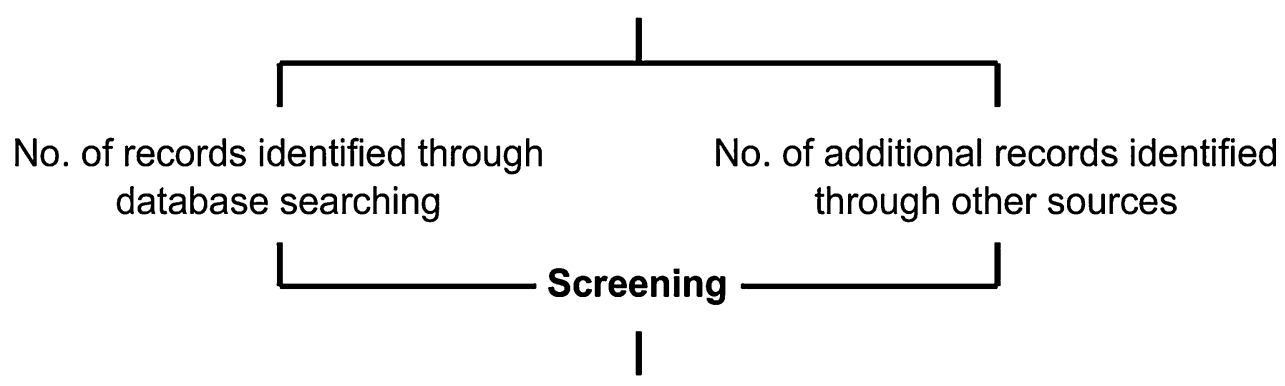

No. of records after duplicates removed

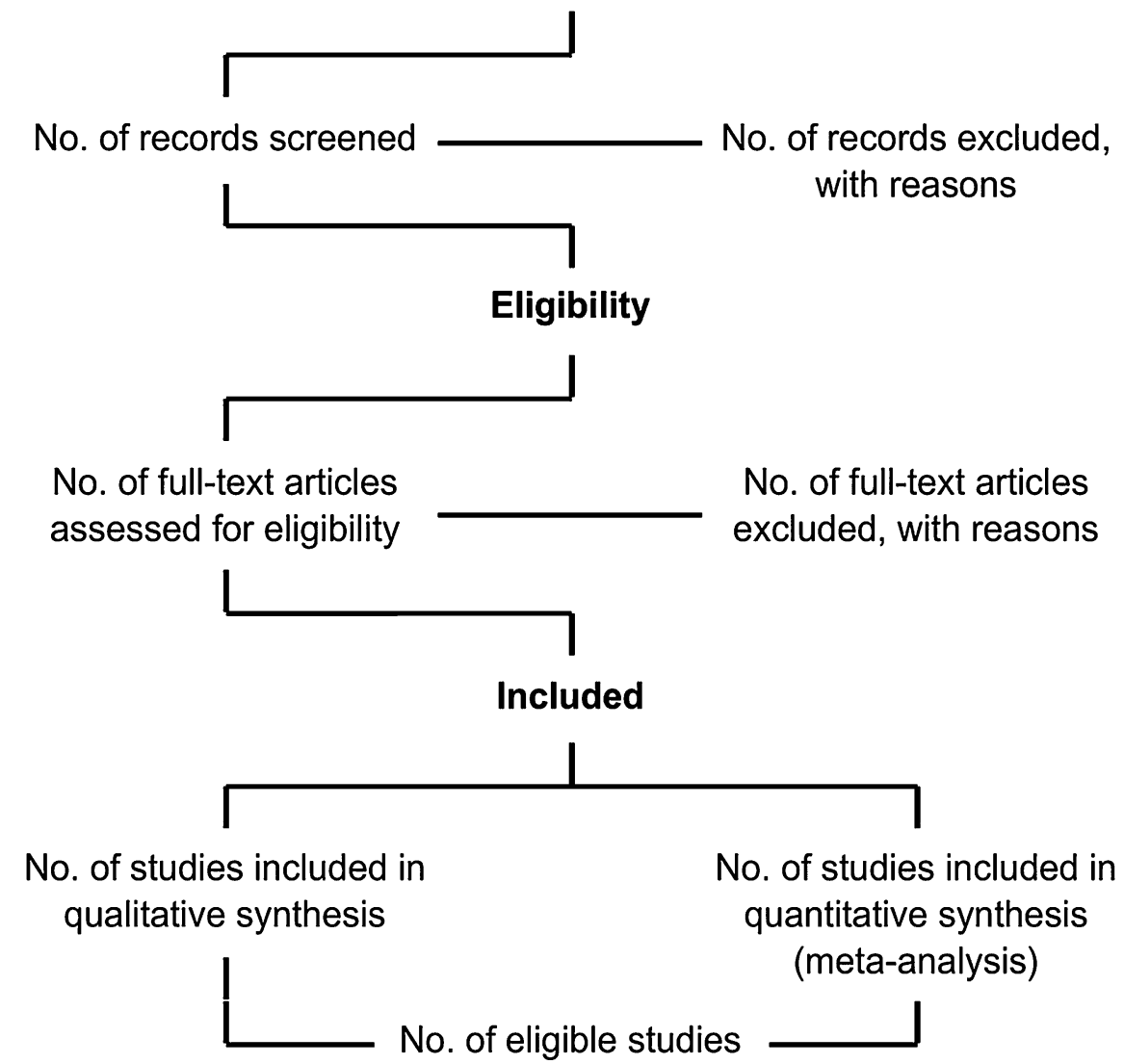

Figure 1. PRISMA flow diagram of study selection

included studies. The paralleled quality assessment methodology will be employed for studies with clinical outcome measures by two additional independent review authors (EP \& AD). Any disagreements in the assessments will be resolved through discussions until a consensus is reached. Otherwise, a third party (JMG or AJG) will be consulted.

\section{Data Extraction and Management}

Data will be abstracted based on the following variables: first author, study location, time points or follow-up periods, study design, setting, types of participants, VAP definitions, comparators, confounders, eligibility criteria, descriptions of educational program including contents of the study module, isolated microorganisms, and learning and clinical outcome measurements.

Two of the review authors (AS \& JA) will abstract the above-mentioned data from each individual report using a structured investigator-made data extraction form, which will be piloted prior to formal data extraction and management. Disagreements between data extractors will be resolved either through a group discussion or the mediation of a third party (JMG or AJG). The review authors will attempt to contact the corresponding authors of the included studies if there are insufficient data that require acquisition. During this period, all requests will be documented and included in the list of studies awaiting assignment. 


\section{Data Analysis and Synthesis}

All relevant patient information, including the data on VAP incidence and microbial colonization, will be pooled using the following descriptive statistics: number, frequency, percentage, mean and standard deviation. These will be calculated and analyzed using the Statistical Package for Social Sciences software (SPSS, Inc, Armonk, NY) for Windows, version 21.0.

For the meta-analysis, the review authors will initially assess the method on how the studies in the analysis were sampled including the types of educational interventions in order to select an appropriate statistical model. If the studies are functionally identical or equivalent and share a common effect size, they will choose a fixed-effect model; otherwise, the random-effects model will be performed.

To compare the learning outcomes with binary data, they will specifically analyze the frequency or proportion of correct response (knowledge) and adherence to VAP prevention strategies using the difference of arcsines transformed proportions (AS) with 95\% confidence interval (CI) for each study. Thereafter, pooled arcsines risk difference with $95 \% \mathrm{Cl}$ will be calculated using either binary fixed-effect inverse variance (IV) model or random-effects DerSimonian-Laird (DL) model as appropriate. For learning outcomes with continuous data, they will calculate the mean difference with $95 \% \mathrm{Cl}$ for each study and combine the effect size using either continuous fixed-effect IV model or random-effects DL model. The review authors will be using OpenMeta[Analyst] for Mac OS X version 0.1503 for the said types of meta-analyses. On the other hand, for clinical data (VAP incidence and mortality) with dichotomous outcomes, they will calculate risk ratios (RR) with $95 \% \mathrm{Cl}$ using either Mantel-Haenszel (M-H) fixed-effect model or random-effects model as applicable.

For data with continuous outcome measures (duration of mechanical ventilation, length of stay, antibiotics and hospitalization costs), the review authors will calculate the mean difference with $95 \% \mathrm{Cl}$ using either IV fixed-effect model or random-effects model as suitable. For these types of meta-analyses, they will be using the Review Manager (RevMan) Software (Copenhagen: Nordic Cochrane Centre, Cochrane Collaboration, 2014) for Mac OS X version 5.3.

The first author (JMG) and the other five review authors (AS, JA, AD, EPJ and AJG) will perform the meta-analysis with the former entering the data into the above-mentioned meta-analysis software packages, and the latter checking the data entry for each outcome measurement to ensure completeness and accuracy.

A predetermined alpha, which is set at $p<0.05$, will be considered significant for all of the above-mentioned analyses. Figure 2 illustrates the planned decision-making algorithm for meta-analysis. In addition, the review authors will assess statistical heterogeneity, perform subgroup and sensitivity analyses, and evaluate publication bias using RevMan. To detect the heterogeneity between studies, they will be applying the Cochran's $Q$ test or $X^{2}$ test with a $p$-value of $<0.10$ as the level of significance. The statistical heterogeneity will be quantified using the $R^{2}$ statistic for both learning and clinical outcomes.

In the present study, the review authors will be applying the recommended threshold of $R$ range valves as described in the Cochrane Handbook for Systematic Reviews of Interventions.[19] The $P$ range values for not important, moderate, substantial and considerable heterogeneity are $0-40 \%, 30-60 \%$, $50-90 \%$ and $75-100 \%$, respectively. If considerable heterogeneity is present and if there is available data, they will investigate the possible differences between the studies and perform subgroup analysis by separating similar study designs, educational interventions, types of ICUs and VAP diagnostic criteria as necessary. Furthermore, they will perform sensitivity analyses by omitting outliers or low-quality evidence (studies with a high risk of bias).

Evidence of publication bias will also be visually investigated using funnel plots for asymmetry. To provide clear presentations and illustrations, the review authors will provide summaries of the findings of included studies using both narrative and tabular syntheses (or in figures as needed). However, if there are methodological issues and significant heterogeneity of samples, they will not perform a meta-analysis, but rather investigate individual study effect size with corresponding $95 \% \mathrm{Cl}$ in a forest plot without calculating the pooled estimates.

\section{Summary of Findings}

The principles of GRADE /Grading of Recommendations, Assessment, Development and Evaluation) system will be used as a guide element to assess the quality of cumulative evidence. [22] Two independent reviewers (JMG \& AS) will 


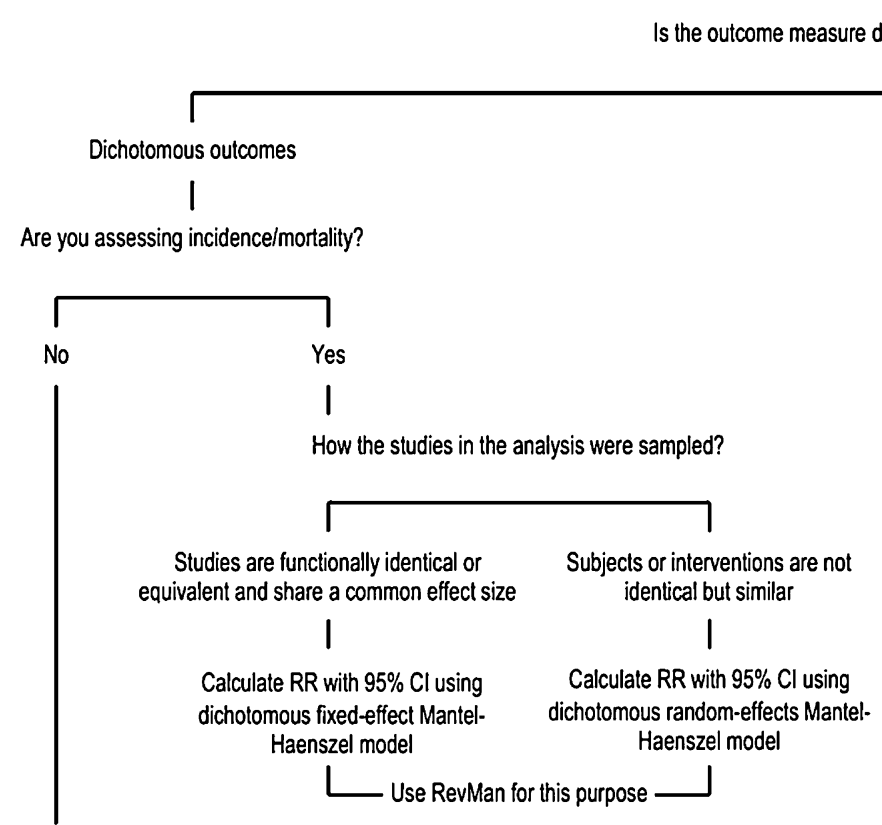

Are you assessing knowledge/adherence?

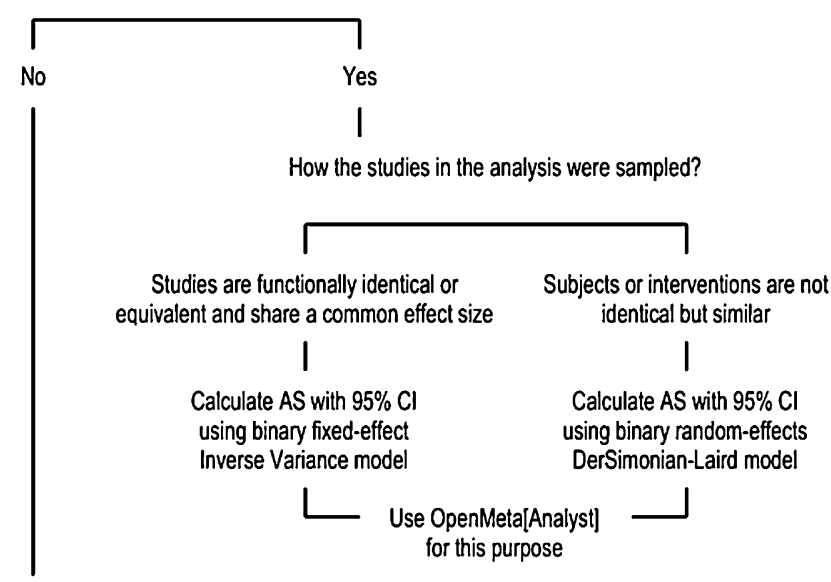

Assess other possible dichotomous outcome measures

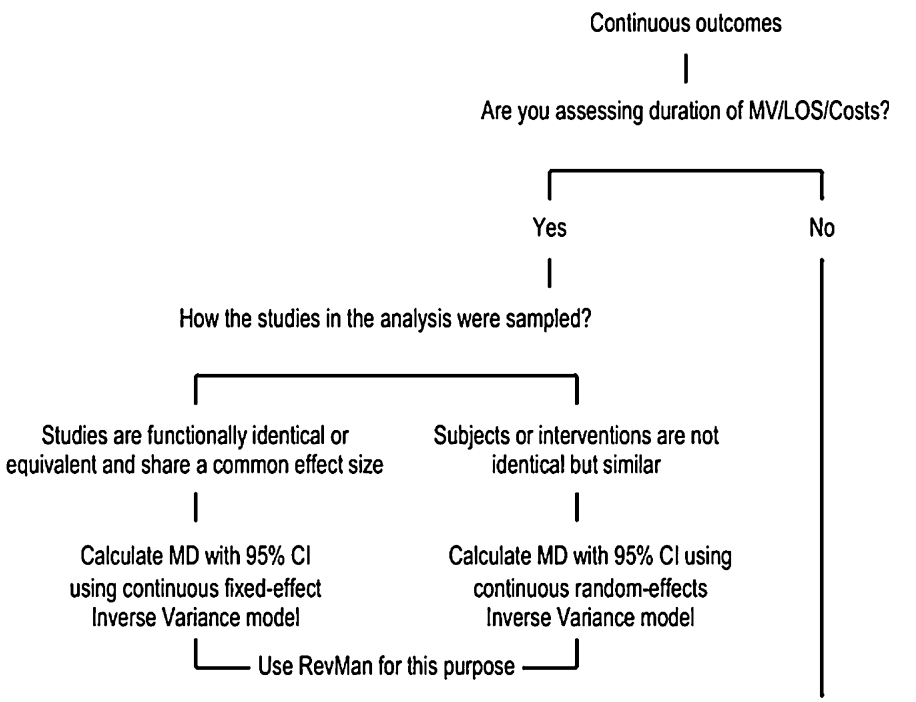

Are you assessing knowledgeladherence?

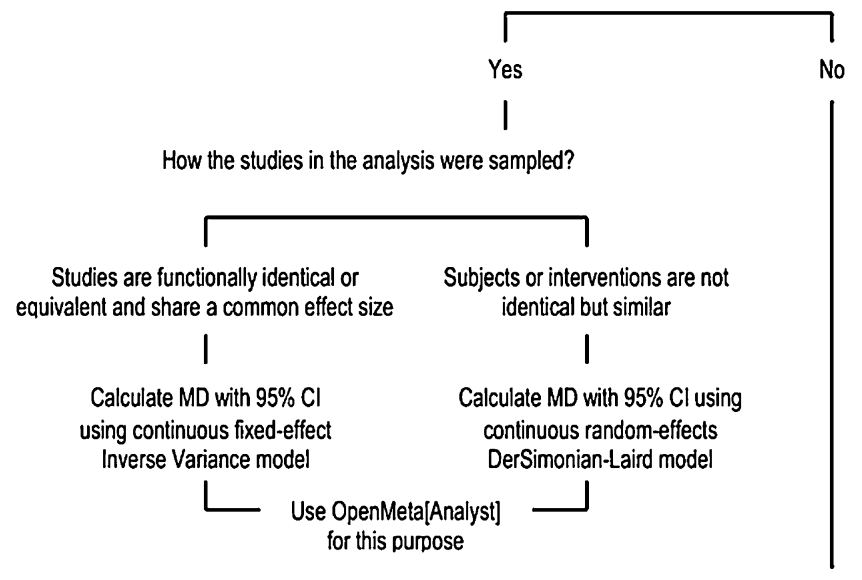

Assess other possible continuous outcome measures

Figure 2. Meta-analysis decision-making algorithm $A S$, arcsines risk difference; $C l$, confidence intervals; $L O S$, length of stay; $M D$, mean difference; $M V$, mechanical ventilation; RR, risk ratio; VAP, ventilator-associated pneumonia.

perform quality assessment measures, considering the risk of bias, inconsistency, indirectness, heterogeneity, imprecision and other considerations for the above-mentioned outcome measures. For each outcome measure, the quality of evidence will be categorized in one of the four levels of grades as recommended: high, moderate, low and very low. Subsequently, they will generate a GRADE evidence table to summarize the review findings. Any disagreements in the grade assignment will be settled through deliberations.

\section{DISCUSSION}

Despite being considered as one of the most preventable types of device-associated nosocomial infections, VAP remains to be a persistent global health concern. Fortunately, this condition can be reduced using a variety of VAP prevention strategies, including well-designed staff education programs. In fact, a number of educational interventions have been revealed to be effective in promoting positive learning and clinical outcomes in intensive and critical care settings. However, there are existing studies that have published conflicting results.

These variations between and among studies may preclude the adoption of an effective VAP educational intervention within intensive and critical care units. Hence, assessing the impact of this kind of intervention on clinicians' learning and patients' outcomes is critical to many areas of healthcare evaluation. This may further suggest the need for a 
systematic review to establish the true effect of the intervention or the association between learning and clinical outcomes.

This current systematic review, therefore, will attempt to summarize the available evidence to address the discordant results between and among studies, and to determine whether or not the institution of educational interventions for VAP prevention is associated with positive outcomes in ICUs.

Insofar as the knowledge of the authors of the present review is concerned, no meta-analysis of association has been previously carried out or conducted on educational intervention for VAP prevention. Although one similar systematic review [10] was conducted, it only addressed research studies that were published between the years 2003 and 2012. Henceforth, an increasing number of similar individual studies have been published in the international body of literature, which warrants further investigation. This current review, therefore, intends to include as many related studies with no date and language restrictions as applicable, considering the unpublished data results, significant heterogeneity between studies and a range of other potential biases. For this reason, the review authors anticipate a number of educational interventions advocating multiple VAP prevention strategies for diverse critically ill populations. They also anticipate a variety of study designs, VAP definitions and patient outcomes. The foregoing relevant issues will be addressed using sound meta-analytic approaches as described above (Figure 2).

On the whole, in this current review, the authors suppose that the results will primarily address the varying and inconsistent approaches to educational interventions in most ICUs, which often lead to uncertainty in improving VAP prevention practices and infection control. Validation of the outcomes will definitely benefit the clinicians, and more importantly, the patients. Furthermore, the study is expected to generate baseline data that will serve as a reference to both ongoing and future clinical practice guidelines and the development of policies, which in turn will help improve healthcare in general. 


\section{Conflict of Interest}

None declared.

\section{ACKNOWLEDGMENT}

The review authors would like to thank Dr. Shirley Landingin for proofreading assistance. This review protocol did not receive any specific grant from funding agencies in the public, commercial, or notfor-profit sectors.

\section{REFERENCES}

1. Safdar N, Dezfulian C, Collard HR, Saint S. Clinical and economic consequences of ventilator-associated pneumonia: A systematic review. Critical Care Medicine. 2005;33(10):2184-93.

2. Hunter JD. Ventilator-associated pneumonia. British Medical Journal. 2012;344:e3325.

3. Coppadoro A, Bittner E, Berra L. Novel preventive strategies for ventilator-associated pneumonia. Critical Care. 2012;16(2):210.

4. Munro N, Ruggiero M. Ventilator-associated pneumonia bundle: Reconstruction for best care. AACN Advanced Critical Care. 2014;25(2):163-75;176-8.

5. Lau AC, So HM, Tang SL, Yeung A, Lam SM, Yan WW, et al. Prevention of ventilator-associated pneumonia. Hong Kong Medical Journal. 2015;21(1):61-8.

6. Danchaivijiitr S, Assanasen S, Apisarnthanarak A, Judaeng $T$, Pumsuwan V. Effect of an education program on the prevention of ventilator-associated pneumonia: A multicenter study. Journal of the Medical Association of Thailand. 2015;88(10):S36-S41.

7. Bouadma L, Mourvillier B, Deiler V, Le Corre B, Lolom I, Régnier $B$, et al. A multifaceted program to prevent ventilator-associated pneumonia: Impact on compliance with preventive measures. Critical Care Medicine. 2010;38(3):789-96.

8. Sinuff T, Muscedere J, Cook DJ, Dodek PM, Anderson W, Keenan SP, et al. Implementation of clinical practice guidelines for ventilator-associated pneumonia: A multicenter prospective study. Critical Care Medicine. 2013;41(1):15-23.

9. Marini AL, Khan R, Mundekkadan S. Multifaceted bundle interventions shown effective in reducing VAP rates in our multidisciplinary ICUs. BMU Quality Improvement Reports. 2016;5(1):u205566-w2278.

10. Jansson $M$, Kääriäinen $M$, Kyngäs $H$. Effectiveness of educational programmes in preventing ventilator-associated pneumonia: A systematic review. Journal of Hospital Infection. 2013;84(3):206-14.

11. Bingham M, Ashley J, De Jong M, Swift C. Implementing a unit-level intervention to reduce the probability of ventilatorassociated pneumonia. Nursing Research. 2010;59/Suppl 1):S40-7.

12. Hamishehkar H, Vahidinezhad M, Mashayekhi SO, Asgharian $\mathrm{P}$, Hassankhani $\mathrm{H}$, Mahmoodpoor A. Education alone is not enough in ventilator associated pneumonia care bundle compliance. Journal of Research in Pharmacy Practice. 2014;3(2):51-5.
13. Khan MS, Siddiqui SZ, Haider S, Zafar A, Zafar F, Khan $R N$, et al. Infection control education: Impact on ventilatorassociated pneumonia rates in a public sector intensive care unit in Pakistan. Transactions of the Royal Society of Tropical Medicine and Hygiene. 2009;103(8):807-1 1.

14. Jam Gatell MR, Santé Roig M, Hernández Vian Ó, Carrillo Santín E, Turégano Duaso C, Fernández Moreno I, et al. Assessment of a training programme for the prevention of ventilator-associated pneumonia. Nursing in Critical Care. 2012;17(6):285-92.

15. Kulvatunyou N, Boonbarwornrattanakul A, Soonthornkit $Y$, Kocharsanee C, Lertsithichai P. Incidence of ventilatorassociated pneumonia (VAP) after the institution of an educational program on VAP prevention. Journal of Medical Association of Thailand. 2007;90(1):89-95.

16. Moola S, Munn Z, Sears K, Sfetcu R, Currie M, Lisy K, et al. Conducting systematic reviews of association (etiology): The Joanna Briggs Institute's approach. International Journal of Evidence-Based Healthcare. 2015;13(3):163-9.

17. Moher D, Shamseer L, Clarke M, Ghersi D, Liberati A, Petticrew $M$, et al. Preferred reporting items for systematic review and meta-analysis protocols (PRISMA-P) 2015 statement. Systematic Review. 2015;4:1.

18. Shamseer L, Moher D, Clarke M, Ghersi D, Liberati A, Petticrew $M$, et al. Preferred reporting items for systematic review and meta-analysis protocols (PRISMA-P) 2015: Elaboration and explanation. British Medical Journal. 2015;349:g7647.

19. Higgins JP, Altman DG, Gøtzsche PC, Jüni P, Moher D, Oxman AD, et al. The Cochrane Collaboration's tool for assessing risk of bias in randomised trials. British Medical Journal. 2011 1;343:d5928.

20. Moher D, Liberati A, Tetzlaff J, Tricco AC, Sampson M, Altman DG. Preferred reporting items for systematic reviews and meta- analyses: The PRISMA statement. British Medical Journal. 2009;339:b2535.

21. Sterne JA, Hernán MA, Reeves BC, Savovic J, Berkman ND, Viswanathan $M$, et al. ROBINS-l: A tool for assessing risk of bias in non-randomised studies of interventions. British Medical Journal. 2016;355:i4919.

22. Guyatt GH, Oxman AD, Kunz R, Falck-Ytter Y, Vist $G E$, Liberati $A$, et al. Rating quality of evidence and strength of recommendations. GRADE: Going from evidence to recommendations. British Medical Journal. 2008;336(7650):1049-51.

(c) (i) Open Access This article is licensed under a Creative Commons Attribution 4.0 International License, which permits use, sharing, adaptation, distribution and reproduction in any medium or format, as long as you give appropriate credit to the original author(s) and the source, provide a link to the Creative Commons license, and indicate if changes were made. The images or other third party material in this article are included in the article's Creative Commons license, unless indicated otherwise in a credit line to the material. If material is not included in the article's Creative Commons license and your intended use is not permitted by statutory regulation or exceeds the permitted use, you will need to obtain permission directly from the copyright holder. To view a copy of this license, visit http://creativecommons.org/licenses/by/4.0/. 